\title{
REVIEW
}

\section{Bone densitometry in children assessed by dual $x$ ray absorptiometry: uses and piffalls}

\section{S Fewtrell, on behalf of the British Paediatric \& Adolescent Bone Group}

Arch Dis Child 2003;88:795-798

The importance of bone growth and mineralisation during childhood for bone health in adult life is increasingly accepted, with both nutritional factors' and exercise ${ }^{2}$ being recognised as influential for normal skeletal development. The appreciation that infancy and childhood are important periods of life for bone development has led to a need for suitable methods for monitoring bone health, both for research purposes and for clinical monitoring of individuals, and hence to an increasing use of bone densitometry in children. This review discusses the role and potential pitfalls of this technique.

See end of article for authors' affiliations

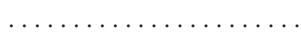

Correspondence to: Dr M Fewtrell, MRC Childhood Nutrition Research Centre, Institute of Child Health, 30 Guilford Street, London WCIN 1 EH, UK m.fewtrell@ich.ucl.ac.uk

Accepted

21 December 2002
$\mathrm{B}$ one densitometry was introduced for use in adults to diagnose and monitor the course of osteoporosis, mainly in post-menopausal women. The most commonly used densitometric technique-dual $x$ ray absorptiometry (DXA)was developed in the late 1980s and is now widely available. Other densitometric methods include quantitative computed tomography (QCT), which has the disadvantage of high radiation doses, making it unsuitable for use in healthy children, and peripheral QCT, which can provide a three dimensional assessment of the structural and geometric properties of the appendicular skeleton with lower radiation exposure. A variety of methods using ultrasound to measure the speed and attenuation of sound through appendicular bone have also been introduced in recent years, although they have not been widely tested in children. This review will concentrate on DXA, which is the technique most commonly used in children in the UK.

Measurements of so-called bone mineral density (BMD) of the hip and lumbar spine have been shown to predict fracture risk in untreated adults, ${ }^{3}$ and the WHO criteria for diagnosing osteoporosis in adults are based on DXA BMD measurements. A T score (defined as the SD score of the observed BMD compared with that of a normal young adult) of $<-1$ SD indicates osteopenia, while a score $<-2.5$ SD defines osteoporosis. ${ }^{4}$ However, $\mathrm{T}$ scores are completely meaningless in children, as they are the equivalent of comparing a child's height to that of an adult. Moreover, while in adult populations DXA measurements have been shown to predict a useful clinical outcome, namely fracture risk, this is not the case for children and younger adults, for whom no such fracture threshold has been defined. These groups are increasingly being referred for DXA scans, yet there are many poten- tial problems with the interpretation of the results, which, in the worst case scenario, may result in erroneous diagnosis and inappropriate treatment. In this article we will review:

- How DXA works

- Problems with the information generated by DXA machines

- Interpretation of DXA scans in children

- How should DXA be used in children?

\section{HOW DXA WORKS}

DXA determines the amount of mineral in a given region by the differential absorption of $x$ rays of two different energies. Using the same differential absorption, it can also take account of the depth and composition of adjacent soft tissue and generate measurements of fat and lean mass. Measurements can be made of the whole body as well as regions such as the lumbar spine, hip, and distal radius. The choice of site may be important because of differences in the proportions of trabecular and cortical bone. Some conditions, such as steroid induced bone loss, affect predominantly trabecular bone, ${ }^{5}$ and may therefore be detected first at the lumbar spine. Others diseases, such as growth hormone deficiency, seem to cause greater deficits in cortical bone and may best be detected by scanning the whole body or distal radius.

Three companies currently manufacture DXA machines (GE Lunar, Hologic, and Norland; the latter being a relatively smaller provider of machines). Each company produces more than one model. From the practical point of view, it is important to determine whether a machine is pencil beam or fan beam. Pencil beam machines acquire data using a small angle beam of $x$ rays that moves across the part being scanned in a rectilinear fashion. Fan beam machines acquire data using a wider angle beam. As a result, scan times are significantly lower with fan beam machines, but the radiation doses are greater, an important issue in children, particularly when collecting reference data from healthy individuals (table 1). In addition, the use of a wide angle beam introduces problems with magnification (see below). The most recent addition to the GE Lunar family, the Prodigy, uses a combination of a narrow angle fan beam with rectilinear scanning to give fast scan times with low radiation doses.

Abbreviations: $\mathrm{BA}$, bone area; $\mathrm{BMC}$, bone mineral content; BMAD, bone mineral apparent density; BMD, bone mineral density; DXA, dual energy $x$ ray absorptiometry; QCT, quantitative computed tomography 


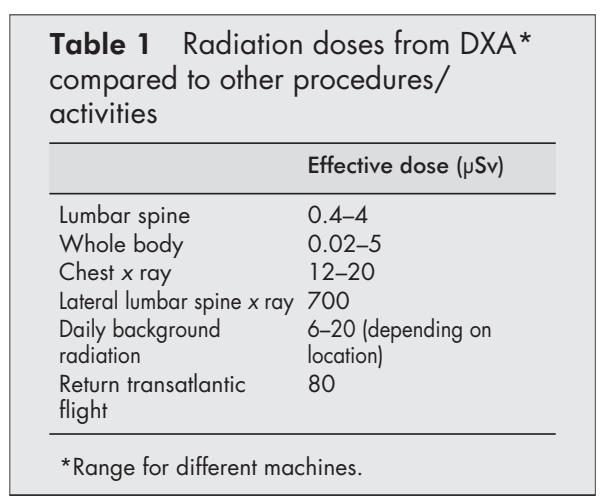

PROBLEMS DURING DATA ACQUISITION

Different machines give different results for a number of reasons relating to both the hardware and software used.

\section{Machine calibration}

GE Lunar machines consistently give BMD values around 16\% higher than Hologic machines, partly because the former are calibrated to ashed bone, while Hologic machines are calibrated to hydroxyapatite. This can cause problems when a patient is scanned using different machines on different occasions, or in research when groups of subjects are scanned in centres with different machines.

\section{Edge detection}

The machine uses an algorithm to detect the edge of the bone being measured. These algorithms vary between machines, and, being designed for use in adults may result in an inability to detect the bone edge in individuals with low bone density. Some machines do have "low density" or "paediatric" software to overcome this problem, but it is important to appreciate that results obtained using these options cannot be directly compared to results obtained from standard or "adult" software. Most machines will, by default, choose what they consider to be the most appropriate software based on the age or weight and height of the subject, although this can generally be over-ridden by the operator. The use of different software options even on the same machine can present problems when scanning an individual longitudinally where they cross from "low" to "normal" or from "paediatric" to "adult" software, or when scanning groups of subjects covering more than one software range. In this situation it may be worth electing to use one software version consistently, even though it may not be the one selected by the machine in all cases. However, care must be taken to ensure that this does not result in insufficient photons being available for analysis in some patients; for example, where a large individual is scanned using "low density" software.

\section{Magnification}

This is a problem with fan beam machines, because of differences in the distance between the bone and the $x$ ray source, depending on the "thickness" of the child. As the machines are designed for adult use, they are programmed to give "correct" results at the distance expected in an average sized adult. However, the projected bone area (BA) and hence bone mineral content (BMC) will vary in smaller (thinner) or larger (thicker) individuals, depending on where the bone is in relation to the $x$ ray source. Theoretically, magnification results in smaller errors in BMD (because errors in both BMC and projected area should to some degree cancel each other out), but it makes interpretation of either BMC or BA measurements alone more difficult- this may be particularly relevant in children.

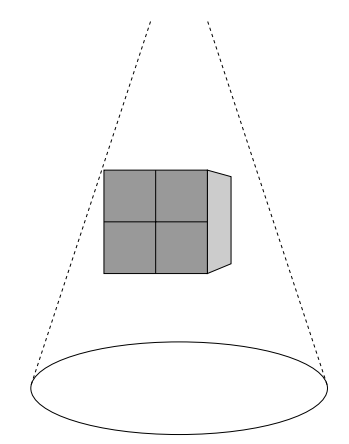

$$
\begin{aligned}
& \text { Mineral weight }=16 \mathrm{~g} \\
& \text { Volume }=8 \mathrm{~cm}^{3} \\
& \text { Projected area }=4 \mathrm{~cm}^{3} \\
& \text { Volumetric density }=2 \mathrm{~g} / \mathrm{cm}^{3} \\
& \text { Areal density }=4 \mathrm{~g} / \mathrm{cm}^{3}
\end{aligned}
$$

Figure 1 Piffalls of areal BMD assessment in children (adapted from Carter et $a^{\prime 13}$ ).

\section{Effect of overlying tissue depth and composition}

It is apparent from in vitro studies using bone phantoms with different overlying depths of fat, water, and air, that both the thickness and composition of tissue surrounding a bone will have an effect on measured BMD. ${ }^{6}$ Unfortunately, these effects seem to be neither linear across the relevant range of tissue thickness, nor consistent between machines. Such effects may be relevant when patients are scanned longitudinally and have changes in body weight, because apparent changes in BMD may partly reflect alterations in body composition. ${ }^{78}$

\section{INTERPRETATION OF DXA SCANS IN CHILDREN}

DXA machines measure bone mineral content (BMC) and bone area (BA), then calculate so-called bone mineral density (BMD) as BMC/BA. It is important to appreciate that BMD is not a true density, but a ratio of the amount of bone and the area scanned. It is thus a two dimensional measurement that is affected by the subject's size. BMD will tend to underestimate bone density in small subjects and overestimate it in larger subjects (fig 1). Most bone densitometers report BMD as a $\mathrm{Z}$ score, related to an age matched (and sometimes gender or ethnic group matched) population. It is important when scanning children to know the source and number of children forming the reference database for the machine used, in order to evaluate how appropriate this is for the child being scanned. The use of sex specific reference data may be particularly important, as Leonard and colleagues ${ }^{9}$ found that the use of non-sex specific reference data resulted in an overdiagnosis of osteopenia in boys.

Although the problem of size effects in bone densitometry are now widely appreciated, there is still no consensus on the most appropriate way to correct results for size, and express them. A number of different approaches have been suggested:

(1) The use of multiple regression analysis simultaneously to adjust BMC for BA, weight, height, and other relevant factors such as age, pubertal status, and calcium intake. ${ }^{10}{ }^{11}$

(2) A modification of (1) in which a staged approach is used, expressing height for age, BA for height, and BMC for BA, to distinguish three possible situations in which reduced bone mass may occur: "short" bones, "narrow" bones, and "thin" bones. $^{12}$

(3) The use of calculated volumetric bone density (bone mineral apparent density, BMAD), in which BMC is adjusted for calculated bone volume rather than bone area. This approach can be used for spine and hip. ${ }^{1314}$ 
Table 2 Conditions in which children may be at increased risk of low bone density and osteoporotic fractures

Chronic inflammatory disease

Systemic long term corticosteroids

Hypogonadism

Osteogenesis imperfecta

Idiopathic juvenile osteoporosis

Prolonged immobilisation

Apparent osteopenia on $x$ ray

(4) A modification of (3) in which bone volume is additionally adjusted for height to correct for body size. ${ }^{15}$

It is likely that different methods may be appropriate for research and for individual patients. However, all methods recognise that $\mathrm{BMC}$ or $\mathrm{BMD}$ measurements need to be interpreted in the context of the child's body size, pubertal stage, and, to a lesser extent, age and ethnic group. In an ideal world, with adequate reference data, it would be possible to input relevant variables such as weight, height, gender, and pubertal stage, together with BMD, and generate a "corrected" BMD value or Z score. However, given the many variables and limitations already discussed, this may not be a realistic proposition.

\section{HOW SHOULD DXA BE USED IN CHILDREN? \\ Research}

In the research situation, DXA may be used to measure the effects of dietary or exercise interventions, often comparing randomised groups. There are fewer problems relating to the need to present the results for an individual in a clinically useful form. However, it is important to be able to interpret changes in BMD independent of those caused by growth. There may be problems if subjects are scanned on different machines or using different software as discussed above.
When reporting results, it is important to specify the machine make, model, and software version used, to enable comparison with other published data.

\section{Clinical}

In the clinical situation, patients are generally scanned either because they are thought to be at risk of low bone density because of their underlying disease or treatment, or to monitor the effects of treatment. Table 2 shows some conditions in which children are at increased risk. It is suggested that performance of a DXA scan should be considered if a child has one of these conditions in conjunction with one of the following symptoms: low trauma or recurrent fractures, back pain, spinal deformity or loss of height, change in mobility status (for example, difficulty walking), or malnutrition. This list is not exhaustive, but covers the conditions most commonly encountered. There are other, rarer, conditions in which bone health may be altered and where assessment by DXA may be indicated (for example, congenital neutropenia, certain inborn errors of metabolism, Ehlers Danlos syndrome, fibrous dysplasia, hypophosphatasia).

It is important to remember that, since many children with chronic disease are small for their age, their BMD measurement will frequently appear to be low as well. Figure 2 illustrates this point with DXA scans from two children. Both have short stature and a similarly low BMD Z score. However, further adjustment for body size, in this case using BMAD as a measure of volumetric bone density, shows a low BMAD Z score in patient 1 , a girl with juvenile arthritis and a history of fragility fractures. In contrast, patient 2 , a boy with growth hormone insensitivity and no bone related symptoms, has a normal BMAD Z score. His low BMD is simply a reflection of his small body size, as indicated by his very low weight $\mathrm{Z}$ score and relatively low bone area and width. These scans illustrate that the result of a DXA scan alone is insufficient for a diagnosis of osteoporosis, and must be considered in conjunction with the clinical picture in order to avoid an inappropriate diagnosis. Particular care must be taken when $\mathrm{T}$ scores are displayed together with WHO definitions of osteoporosis that

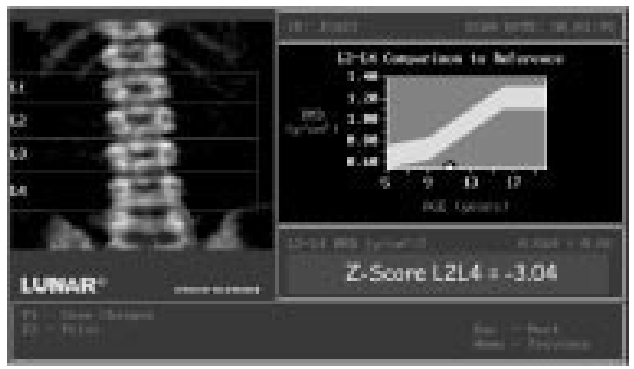

Case 1

$\begin{array}{ll}\text { Diagnosis } & \text { Juvenile arthritis with fragility fractures } \\ \text { Sex } & \text { Female } \\ \text { Age } & 11.5 \text { years } \\ \text { BMD Z score } & \mathbf{- 3 . 0} \\ \text { Height Z score } & -5.2 \\ \text { Weight Z score } & -2.7 \\ \text { BMC }(\mathrm{g}) & 10.96 \\ \text { BA }\left(\mathrm{cm}^{2}\right) & 17.54 \\ \text { Bone width }(\mathrm{cm}) & 3.1 \\ \text { BMAD Z score } & -2.2\end{array}$

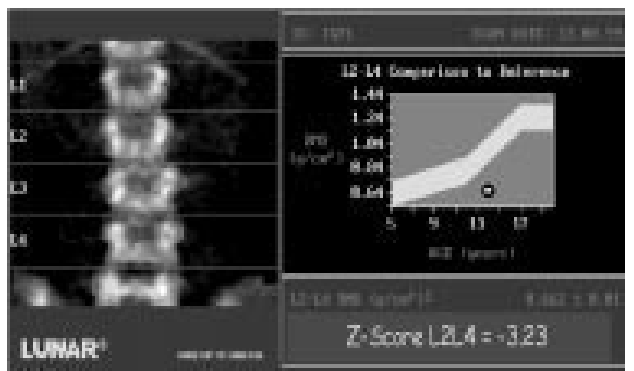

Case 2

Growth hormone insensitivity syndrome, no bone symptoms Male

14.7 years

$\mathbf{- 3 . 2}$

$-5.0$

$-4.7$

13.50

20.38

2.8

$-0.7$

Figure 2 Examples of the effect of body size on $B M D$. *BMAD was calculated using the method of Kroger et al. ${ }^{14} \mathrm{BMAD} Z$ scores were calculated as follows. Case 1: BMAD Z score $=(0.234-0.314) / 0.035=-2.3$, where the mean $(S D)$ value for an 11 year old Caucasian female is $0.314(0.035)$. Case 2: $B M A D Z$ score $=(0.300-0.320) / 0.030=-0.7$, where the mean (SD) value for a 14 year old Asian boy is 0.320 (0.030). Reference population from the same geographical area as the subjects, and matched for age, gender, and ethnic group. Note that BMAD in boys lags behind that of girls during adolescence because of the later onset of puberty; thus the values for an 11 year old girl and 14 year old boy are very similar. 
are not appropriate in children. Ideally, the $\mathrm{T}$ score display should be switched off.

It is important to be aware that vertebral crush fractures may result in an artefactually high BMD value for the affected vertebra. This pitfall can be avoided by inspecting both the image and BMD for each vertebra scanned, and comparing vertebral size, rather than simply looking at the total lumbar spine BMD. In addition, certain diseases that affect vertebral morphology may also affect BMD since flattening or widening of the vertebra as seen, for example, in thalassaemia may result in increased measured BMD despite identical volumetric bone mass.

At present, given the difficulties discussed, a child may most appropriately act as his or her own control, with serial scans to monitor progress. Scan intervals of less than six months should only be considered in special situations such as monitoring the response to a pharmacological intervention, and for most patients annual scans should suffice. This approach will mean that the clinical and prognostic value of DXA should become apparent over the next few years, putting us in a better position to define the groups of children who most benefit from the investigation as well as to improve the prognostic value of a single measurement. It will also allow us to establish whether low BMD measurements that are considered to be a reflection of small body size have consequences for later bone health.

At present, we would recommend that "clinical" DXA scans should not be performed outside centres with a clinical team with a specific interest and expertise in bone densitometry in children. Alternatively, if scans are performed elsewhere, clinicians should consider consulting one of these centres for advice, particularly if they are considering starting or changing treatment on the basis of a scan result

\section{ACKNOWLEDGEMENTS}

We are grateful to Professor Isky Gordon, Department of Radiology, Great Ormond Street Hospital NHS Trust, for his helpful comments on the manuscript.

\section{Authors' affiliations}

M S Fewtrell, on behalf of the British Paediatric \& Adolescent Bone Group, MRC Childhood Nutrition Research Centre, Institute of Child Health, London, and Great Ormond Street Hospital NHS Trust, London, UK

British Paediatric \& Adolescent Bone Group (BPABG): S F Ahmed, Consultant Paediatric Endocrinologist, Royal Hospital for Sick Children, Glasgow; J Allgrove, Consultant Paediatric Endocrinologist, Newham General, Royal London and Great Ormond Street Hospitals, London;
N J Bishop, Professor of Paediatric Bone Disease, Sheffield Children's Hospital; N J Crabtree Clinical Scientist, Department of Medical Physics, Queen Elizabeth Hospital, Birmingham; M S Fewtrell, Clinical Scientist \& Honorary Consultant Paediatrician, Institute of Child Health \& Great Ormond Street Hospital, London; J W Gregory, Senior Lecturer in Paediatric Endocrinology, University of Wales College of Medicine, Cardiff; Z Mughal, Consultant Paediatrician, St Mary's Hospital, Manchester; S P Ryan, Consultant Paediatrician, Alder Hey Children's Hospital, Liverpool; N J Shaw* , Consultant Paediatric Endocrinologist, Birmingham Children's Hospital; C M Smith, Research Associate, Bone Metabolism Group, University of Sheffield; K Ward, Clinical Radiology, Imaging Science \& Biomedical Engineering, University of Manchester

*Contact for BPABG

\section{REFERENCES}

1 Eastell R, Lambert H. Diet and healthy bones. Calcif Tissue Int 2002;70:400-4.

2 Morris FL, Naughton GA, Gibbs JL, et al. Prospective ten-month exercise intervention in premenarcheal girls: positive effects on lean and bone mass. J Bone Miner Res 1997; 12:1453-62.

3 Cummings SR, Marcus R, Palermo L, et al. Does estimated volumetric bone density of the femoral neck improve the prediction of hip fracture? A prospective study of the Osteoporotic Fracture Research Group. J Bone Miner Res 1994;9:1429-32.

4 WHO. Assessment of fracture risk and its application to screening for postmenopausal osteoporosis: report of a WHO study group. WHO Technical Report Series 843. Geneva: WHO, 1994

5 Bianchi ML. Glucocorticoids and bone: some general remarks and some special observations in pediatric patients. Calcif Tissue Int 2002;70:384-90

6 Laskey MA, Prentice A. Comparison of adult and paediatric spine and whole body software for the Lunar dual energy $\mathrm{X}$-ray absorptiometer. $\mathrm{Br} \mathrm{J}$ Radiol 1999:72:967-76.

7 Bolotin HH. A new perspective on the causal influence of soft tissue composition on DEXA-measured in vivo bone mineral density. J Bone Miner Res 1998; 13:1739-46

8 Tothill $\mathbf{P}$, Laskey MA, Orphanidou $\mathrm{Cl}$, et al. Anomolies in dual energy $X$-ray absorptiometry measurements of total-body bone mineral during weight change using Lunar, Hologic and Norland instruments. BrJ Radiol 1999;72:661-9.

9 Leonard MB, Propert KJ, Zemel BS, et al. Discrepancies in pediatric bone mineral density reference data: potential for misdiagnosis of osteopenia. J Pediatr 1999:135: 182-8.

10 Prentice A, Parsons TJ, Cole TJ. Uncritical use of bone mineral density in absorptiometry may lead to size-related artefacts in the identification of bone mineral determinants. Am J Clin Nutr 1995;60:837-42.

11 Warner JT, Cowan FJ, Dunstan FD, et al. Measured and predicted bone mineral content in healthy boys and girls aged 6-18 years: adjustment for body size and puberty. Acta Paediatr 1998;87:244-9.

12 Molgaard C, Thomsen GL, Prentice A, et al. Whole body bone mineral content in healthy children and adolescents. Arch Dis Child 1997:76:9-15.

13 Carter DR, Bouxsein ML, Marcus R. New approaches for interpreting projected bone densitometry data. J Bone Miner Res 1992;7:137-45.

14 Kroger $\mathbf{H}$, Kotaniemi A, Vainio $P$, et al. Bone densitometry of the spine and femur in children by dual-energy $x$-ray absorptiometry. Bone Miner 1992; 17:75-85.

15 Smith CM, Coombs RC, Gibson AT, et al. Approaches to adjusting bone mineral content for bone size in children. Calcif Tissue Int 2002;70:370(A) 